\title{
TRIB1 regulates LDL metabolism through CEBP $\alpha$-mediated effects on the LDL receptor in hepatocytes
}

\author{
Katherine Quiroz-Figueroa, ${ }^{1}$ Cecilia Vitali, ${ }^{1}$ Donna M. Conlon, ${ }^{1}$ John S. Millar, ${ }^{1}$ John W. Tobias, ${ }^{2}$ Robert C. Bauer, ${ }^{1}$ Nicholas J. Hand, ${ }^{2,4}$ \\ and Daniel J. Rader 1,2,3,4 \\ 'Division of Translational Medicine and Human Genetics, Department of Medicine, ${ }^{2}$ Department of Cenetics, ${ }^{3}$ Department of Pediatrics, and ${ }^{4}$ Institute for Translational Medicine and Therapeutics, Perelman \\ School of Medicine, University of Pennsylvania, Philadelphia, Pennsylvania, USA
}

\begin{abstract}
Genetic variants near the TRIB1 gene are highly significantly associated with plasma lipid traits and coronary artery disease. While TRIB1 is likely causal of these associations, the molecular mechanisms are not well understood. Here we sought to investigate how TRIB1 influences low density lipoprotein cholesterol (LDL-C) levels in mice. Hepatocyte-specific deletion of Trib1 (Trib1 ${ }^{\text {hhep }}$ ) in mice increased plasma cholesterol and apoB and slowed the catabolism of LDL-apoB due to decreased levels of LDL receptor (LDLR) mRNA and protein. Simultaneous deletion of the transcription factor CCAAT/enhancer-binding protein alpha (CEBP $\alpha$ ) with TRIB1 eliminated the effects of TRIB1 on hepatic LDLR regulation and LDL catabolism. Using RNA-seq, we found that activating transcription factor 3 (Atf3) was highly upregulated in the livers of Trib $1^{\text {Ahep }}$ but not Trib1 ${ }^{\text {Ahep }}$ Cebpa ${ }^{\text {Ahep }}$ mice. ATF3 has been shown to directly bind to the CEBP $\alpha$ protein, and to repress the expression of LDLR by binding its promoter. Blunting the increase of ATF3 in Trib1 ${ }^{\text {hep }}$ mice reduced the levels of plasma cholesterol and partially attenuated the effects on LDLR. Based on these data, we conclude that deletion of Trib1 leads to a posttranslational increase in CEBP $\alpha$, which increases ATF3 levels, thereby contributing to the downregulation of LDLR and increased plasma LDL-C.
\end{abstract}

\section{Introduction}

The TRIB1 gene, which encodes Tribbles homolog 1 protein (TRIB1), has been suggested as the causal effector transcript underlying the genome-wide association study (GWAS) signal at human chromosomal locus 8q24 for a remarkable number of cardiometabolic traits, including plasma lipids and lipoproteins, hepatic steatosis, adiponectin levels, and coronary artery disease (1-8). The tribbles gene was first identified in Drosophila as a regulator of cell division and morphogenesis and was shown to regulate the proteins encoded by string and slbo, the Drosophila homologs of the dual phosphatase CDC25A and CEBP $\alpha$, respectively (9-11). In humans, TRIB1 encodes a highly conserved pseudokinase that functions as a signal transduction pathway modulator and scaffolding protein (12-14), including interacting with the E3 ubiquitin ligase COP1 (also known as RFWD2) to target the transcription factors $\mathrm{CEBP} \alpha$ and $\mathrm{CEBP} \beta$ for ubiquitination and proteasomal degradation, a process that has been primarily explored in myeloid cell differentiation (15-18). While other proteins, including SAP18-Sin3A (19), HNF4A (20), ChREBP,

Authorship note: NJH and DJR contributed equally to this work. Conflict of interest: DJR serves on the Scientific Advisory Boards of Alnylam, Novartis, Pfizer, and Verve Therapeutics, and is a Founder of VascularStrategies and Staten Biotechnologies.

Copyright: ( 2021, American Society for Clinical Investigation.

Submitted: December 9, 2020; Accepted: September 21, 2021;

Published: November 15, 2021.

Reference information: / Clin Invest. 2021;131(22):e146775.

https://doi.org/10.1172/JCl146775. and NFKB (21), have been suggested to be putative targets of TRIB1, regulation of the COP1-CEBP $\alpha$ axis remains the most fully described function of TRIB1.

Since the establishment of a genetic link between TRIB1 and cardiometabolic traits, limited investigation of its role in the liver has been performed. We previously reported that Trib1 overexpression in mouse liver decreased plasma lipids, hepatic de novo lipogenesis, and very low-density lipoprotein (VLDL) production and that Trib1 hepatic deletion had the opposite effects $(22,23)$. Furthermore, Trib1 deletion markedly increased hepatic levels of CEBP $\alpha$ protein, which helped explain the increased de novo lipogenesis and hepatic lipid phenotypes (23). However, questions regarding the physiological and molecular mechanism by which TRIB1 specifically regulates plasma LDL-C metabolism remained unanswered.

\section{Results}

Hepatocyte-specific deletion of Trib1 increased plasma lipids and lipoprotein levels. To assess the hepatic effects of Trib1 deletion on LDL-C, we injected Trib1 ${ }^{f l f l}$ mice with AAV8-TBG-Cre to induce hepatocyte-specific deletion of Trib1 (Trib1 ${ }^{\text {shep }}$ ) and compared them with control mice WT for Trib1 (Trib1 ${ }^{W T}$ ) receiving the same AAV treatment. Trib1 ${ }^{\text {shep }}$ male mice had a greater than $95 \%$ reduction in liver Trib1 mRNA after AAV8-TBG-Cre injection on chow diet (Supplemental Figure 1A; supplemental material available online with this article; https://doi.org/10.1172/JCI146775DS1) and on Western type diet (WTD) (Supplemental Figure 1B), observations also noted in female mice (Supplemental Figure 1, C and D). The residual Trib1 mRNA is due to its expression in nonparen- 


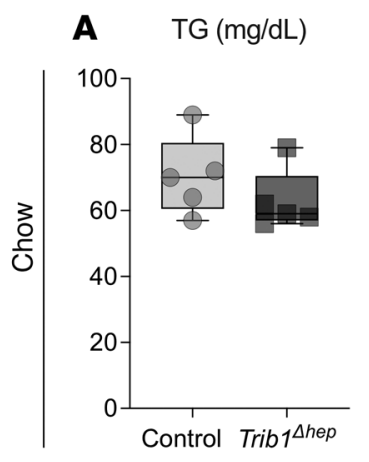

E

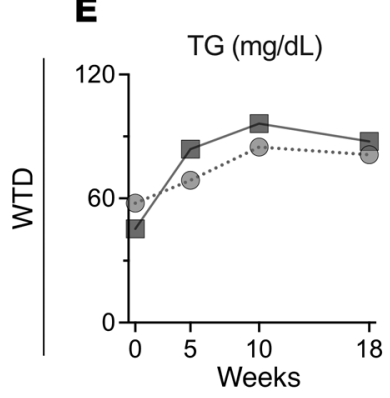

I

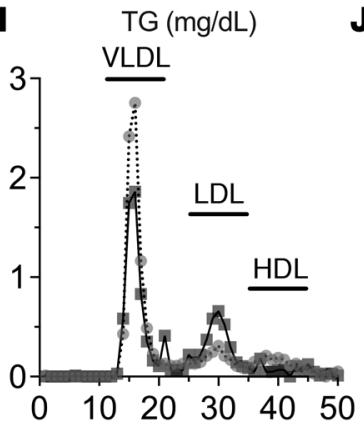

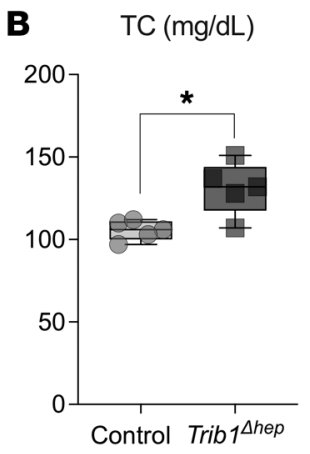

F $\quad \mathrm{TC}(\mathrm{mg} / \mathrm{dL})$

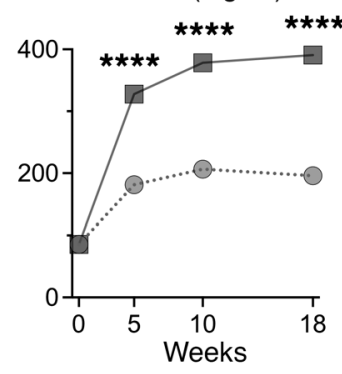

$\mathbf{J}$

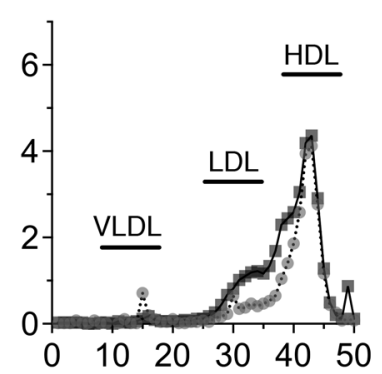

FPLC - chow
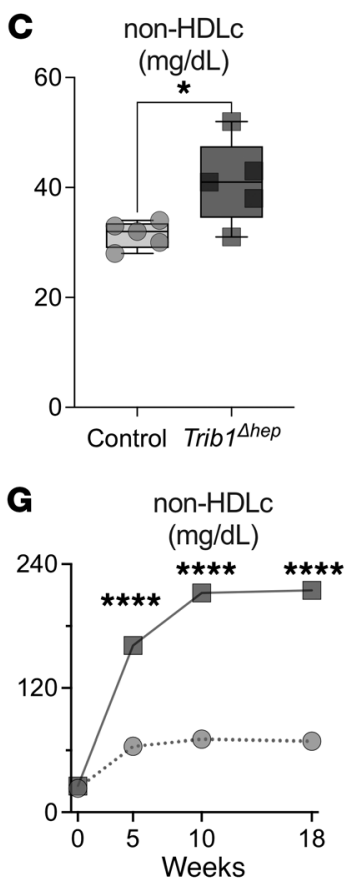

K

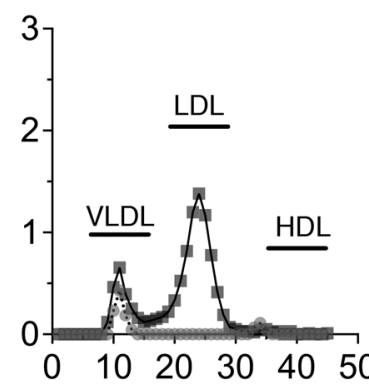

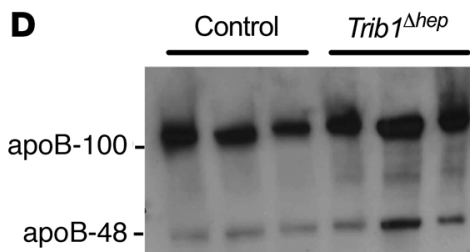

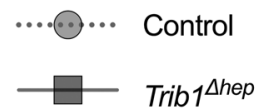

H

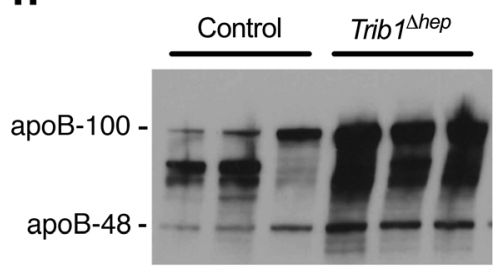

L $\quad \mathrm{TC}(\mathrm{mg} / \mathrm{dL})$

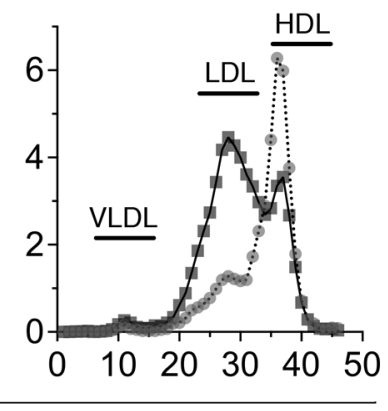

PLC - WTD

Figure 1. Hepatic deletion of Trib1 increases total and non-HDL cholesterol, LDL-TG, LDL-TC, and circulating apoB. (A-C) Plasma triglycerides, total cholesterol, and non-HDL cholesterol levels in chow-fed male mice 4 weeks after deletion. Box plots indicate median and 25th and 75 th percentiles, with whiskers extending to minimum and maximum values. Symbols indicate single values $(n=5)$ of a representative experiment. Results were confirmed in 5 independent cohorts at a variety of time points after deletion. Significance was determined using 2-tailed, unpaired Student's $t$ test $\left({ }^{*} P \leq 0.05\right)$. (D) Immunoblot of apoB protein in plasma from chow-fed mice 4 weeks after deletion. Equal volumes of plasma were loaded in each well. Blots were replicated in 2 independent cohorts in both chow- and WTD-fed mice. (E-G) Plasma triglycerides, total cholesterol, and non-HDL cholesterol levels at selected time points over 18 weeks of WTD feeding $(n=8)$. Representative experimental data are expressed as mean \pm SEM and were confirmed in 2 independent cohorts. Significance was determined using 2-way ANOVA with Tukey's multiple comparisons test $\left({ }^{* * *} P \leq 0.0001\right)$. (H) Immunoblots of apoB protein in plasma from WTD-fed mice for 11 weeks, ran as image D. (I-L) Fast protein liquid chromatography (FPLC) was performed on pooled plasma from chow-fed mice for 6 weeks and WTD-fed mice for 12 weeks to separate lipoproteins based on their size. $x$ axis: fraction number. Triglycerides (I and $\mathbf{K}$ ) and total cholesterol ( $\mathbf{J}$ and $\mathbf{L}$ ) concentrations were measured in all fractions. FPLC results were confirmed in 3 independent cohorts in chow-fed mice and 2 independent cohorts in WTD-fed mice.

chymal cells, where Trib1 is not deleted due to the high specificity of the AAV8-TBG vector for hepatocytes. Trib1 ${ }^{\text {shep }}$ male mice had no statistically significant change in triglyceride (TG) levels on either chow or WTD (Figure 1, A and E) but had markedly increased total cholesterol (TC), non-high-density lipoprotein cholesterol (non-HDL-C) levels and plasma apoB protein (Figure $1, \mathrm{~B}-\mathrm{D}$ ). The dysregulation of plasma lipid traits and apoB was markedly exacerbated by WTD feeding (Figure 1, F-H). Lipoprotein fractionation of plasma from male Trib1 ${ }^{\text {shep }}$ mice revealed that the increase in cholesterol was specific to LDL on both chow (Fig- ure 1, I and J) and WTD (Figure 1, K and L). Trib1 ${ }^{\text {shep }}$ female mice had broadly similar but less pronounced plasma lipid phenotypes on both chow and WTD (Supplemental Figure 2).

Trib $^{\text {shep }}$ mice exhibit delayed apoB-lipoprotein clearance due to decreased LDLR levels. To investigate the mechanism responsible for the elevated LDL-C in Trib1 ${ }^{\text {shep }}$ mice, we injected ${ }^{125} \mathrm{I}$-LDL-apoB into Trib1 $1^{\text {shep }}$ and control mice and traced its clearance from the plasma over 24 hours. Male Trib1 ${ }^{\text {shep }}$ mice exhibited markedly slower LDL-apoB catabolism, both on a chow diet (Figure 2A) where the fractional catabolic rate (FCR) of LDL-apoB was 2.1-fold slower in 

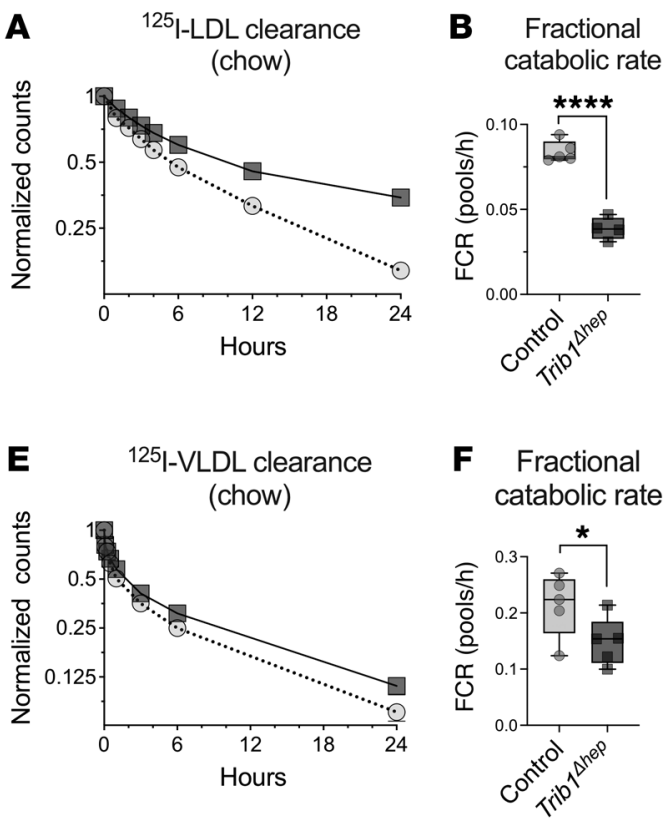

\section{Fractional catabolic rate}
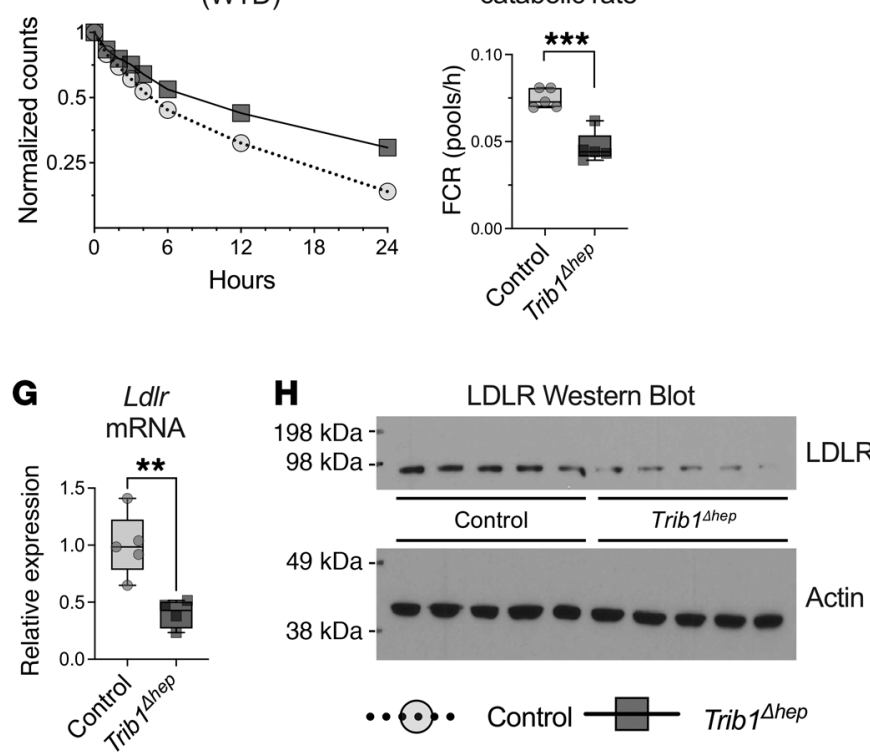

Figure 2. Hepatic deletion of Trib1 impairs LDL clearance due to decreased LDLR mRNA and protein. (A and B) LDL clearance in chow-fed mice 8 weeks after AAV injection $(n=5)$ and $(\mathbf{C}$ and $\mathbf{D})$ in mice fed WTD for 19 weeks (24 weeks after AAV injection) (A-D) Mice were injected with 125l-radiolabeled LDL isolated from human plasma. Clearance of 125I-LDL was determined by measuring residual 125 lactivity at different time points after injection (from 2 minutes to 24 hours). Residual 125 activity is expressed as fraction of the total 1251 activity, 2 minutes after injection. (B and D) Fractional catabolic rate (FCR) of LDL from $\mathbf{A}$ and $\mathbf{C}$, calculated from the reciprocal of the area under a fitted biexponential curve, and it represents fraction of LDL cleared per hour. Results were confirmed in an independent cohort in chow-fed mice and 2 independent cohorts in WTD-fed mice. (E) VLDL clearance in chow-fed male mice 8 weeks after AAV injection. Mice were injected with 125I-radiolabeled human VLDL, and the total remaining counts were normalized to the injected dose at 1 minute after injection of 125IVLDL. (F) FCR of 125I-VLDL. (C) Hepatic transcript levels of LDLR in chow-fed male Trib1 ${ }^{\text {thep }}$ mice relative to control mice 8 weeks after AAV injection $(n=5)$. The relative quantity of mRNA normalized to the combined mean Ct of Mrlp19, Ywhaz, and Ipo8, and expressed relative to the mean of the control group. (H) Hepatic protein levels of LDLR and $\beta$-actin in control and Trib1 ${ }^{\text {shep }}$ mice $(n=5)$. Both LDLR mRNA and protein levels changes were confirmed in 3 independent cohorts. (A, C, and D) Data are expressed as mean \pm SEM for the experimental group. (B, D, F, and G) Box plots indicate median and 25th and 75 th percentiles, with whiskers extending to minimum and maximum values. Symbols indicate individual values. Significance was determined by 2 -tailed, unpaired Student's $t$ test $\left.{ }^{* *} P \leq 0.01,{ }^{* *} P \leq 0.001,{ }^{* * *} P \leq 0.0001\right)$.

Trib1 ${ }^{\text {shep }}$ mice than WT mice (Figure 2B), as well as on WTD (Figure 2C) where the FCR was 1.6-fold slower than WT mice (Figure 2D). Similar reductions in LDL catabolism were noted in female Trib1 $1^{\text {she }}$ mice, though not to the same degree as in male mice (Supplemental Figure 3, A-D). We also injected Trib1 ${ }^{\text {sep }}$ and control mice with ${ }^{125} \mathrm{I}-\mathrm{VLDL}-\mathrm{apoB}$ and traced its clearance over 24 hours. Trib1 $1^{\text {she }}$ mice had a significant reduction in the rate of VLDL-apoB catabolism compared with control mice (Figure 2, E and F). Thus, Trib1 ${ }^{\text {she }}$ mice have substantially slower turnover of both LDL-apoB and VLDL-apoB compared with control mice, contributing to the accumulation of plasma apoB-containing lipoproteins.

The hepatic LDL receptor (LDLR) is a key regulator of LDLapoB catabolism and genetic variants that reduce LDLR expression or function lead to elevated plasma LDL-C levels (24). Both male and female Trib1 $1^{\text {shep }}$ mice on chow diets had significantly reduced hepatic abundance of Ldlr mRNA compared with control mice (Figure $2 \mathrm{G}$ and Supplemental Figure 3E), as well as reduced levels of LDLR protein (Figure 2H and Supplemental Figure 3F). Thus, deletion of Trib1 in mouse hepatocytes resulted in substantial downregulation of the LDLR, providing a molecular basis for the impaired clearance of apoB-containing lipoproteins.

The delayed apoB-lipoprotein clearance in Trib ${ }^{\text {shep }}$ mice is dependent on the LDLR. To determine whether the effects of Trib1 deletion on the clearance of apoB-containing lipoproteins were medi- ated solely by downregulation of the LDLR, we generated Trib $1^{\text {th }}$ ${ }^{f} \mathrm{~L} d l r-\mathrm{KO}$ mice and injected them with AAV8-TBG-Cre or AAV8TBG-Null. Hepatic Trib1 mRNA levels were decreased by more than $95 \%$ in Trib1 ${ }^{\text {shep }} L d l r-K O$ mice, and Ldlr mRNA and protein were undetectable in both groups compared with controls (Supplemental Figure 4, A and B). We performed LDL-apoB and VLDLapoB kinetic studies in these 2 groups of mice as well as control mice. As expected, the turnover of both LDL-apoB and VLDLapoB was markedly slower in Trib1 WT Ldlr-KO mice compared with control mice due to loss of the LDLR (Figure 3, A-D). However, loss of Trib1 in the absence of the LDLR had no further effect on clearance of LDL-apoB and VLDL-apoB (Figure 3, A-D). These data indicate that the effect of Trib1 deletion on apoB-containing lipoprotein clearance is dependent on the LDLR.

Trib1 ${ }^{\text {shep }}$ mice have increased steady-state plasma lipids due to increased apoB-lipoprotein production. We also measured plasma lipids and found that deletion of Trib1 in hepatocytes in the absence of LDLR further increased plasma TG, TC, and non-HDL-C compared with $L d l r-K O$ mice with normal hepatocyte TRIB1 (Figure 3, $E-G)$. Fractionation of plasma by FPLC revealed that TC (Figure $3 \mathrm{H}$ ) and TG (Supplemental Figure 4C) were increased in both the VLDL and LDL fractions. These results indicate that Trib1 deletion also increases plasma lipids in a mechanism that is independent of its regulation of the LDLR and apoB clearance. 

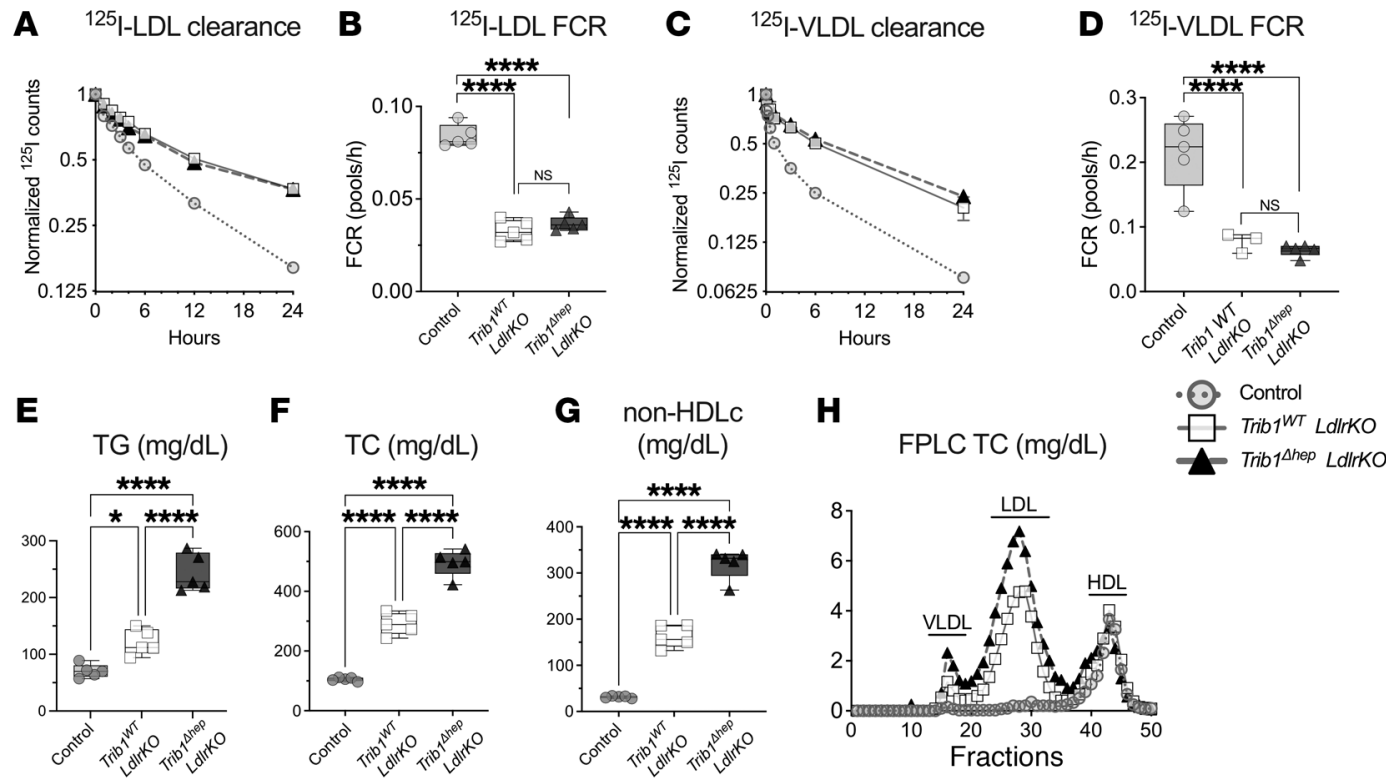

Figure 3. The delayed apoB-lipoprotein clearance in Trib1 ${ }^{\text {thep }}$ mice is dependent on the LDLR but loss of TRIB1 further increases steady-state plasma lipids in the absence of LDLR. (A) 125I-LDL clearance in chow-fed male mice 8 weeks after AAV injection $(n=5)$. (B) FCR from A. (C) 125I-VLDL clearance in chow-fed male mice 8 weeks after AAV injection. (D) FCR from panel C. Results were confirmed in an independent cohort. (E-C). Plasma triglycerides, total cholesterol, and non-HDL cholesterol levels in chow-fed male mice 6 weeks after deletion. (H) FPLC of total cholesterol performed on pooled plasma from chow-fed mice for 6 weeks after AAV8-TBG-Cre injection. Results were confirmed in 3 independent cohorts. (A and $\mathbf{C}$ ) Data are expressed as mean \pm SEM for the experimental group. (B, D, E, F, and $\mathbf{C}$ ) Box plots indicate median and 25th and 75th percentiles, with whiskers extending to minimum and maximum values. Symbols indicate individual values. Significance was determined by 1-way ANOVA with Tukey's multiple correction test (**** $P \leq 0.0001$ ).

We measured production of newly synthesized, ${ }^{35} \mathrm{~S}-\mathrm{Met} /$ Cys-labeled apoB in mice that had been injected with pluronic to inhibit plasma lipolysis. Our results show that Trib1 ${ }^{\Delta h e p}$ mice have markedly increased apoB production despite no difference in $\mathrm{TG}$ secretion (Figure 4, A and B). We also measured apoB secretion in the context of the $L d l r-K O$ background and observed the same results (Figure 4, C and D). The increase in apoB secretion without an increase in TG secretion suggested that a relatively TG-poor, apoB-containing lipoprotein is being secreted by the Trib1 ${ }^{\text {thep }}$ hepatocytes. To test this, we pooled postpluronic plasma 2 hours after injection and performed sucrose density ultracentrifugation to separate the lipoproteins based on their density. This experiment showed a shift of the newly synthesized apoB from VLDL toward the LDL density fractions in the Trib1 $1^{\text {shep }}$ mice compared with control mice (Figure 4E), consistent with the secretion of denser apoB-containing lipoproteins. Consistent with the higher steady-state apoB levels noted by immunoblot in Trib1 ${ }^{\text {shep }}$ mice (Figure 1, D and H) apoB levels were higher throughout the gradient in the Trib1 ${ }^{\text {shep }}$ sample (Figure 4, E and F). We normalized the apoB signal intensity per fraction to the total signal per sample and noted a rightward shift of the fractional distribution peak that indicated an increase in the relative density of the apoB lipoprotein particles in the Trib1 ${ }^{\Delta h e p}$ mouse plasma that reflects more numerous lipid-poor particles (Figure 4G).

Because ER stress is known to result in increased secretion of apoB lipoprotein particles $(25,26)$, we assessed pEIF2a, a known marker of ER stress, and found it to be markedly increased in $T_{\text {Trib1 }}{ }^{\text {shep }}$ mice independent of L $d l r$ genotype (Supplemental Figure 4, C-E). We conclude that hepatocyte-specific Trib1 deletion results in increased production of apoB particles that are relatively lipid-poor and therefore denser, exacerbating the increase in plasma LDL-C and apoB levels beyond the contribution of delayed LDL-apoB clearance.

The effects of Trib1 ${ }^{\text {shep }}$ on plasma lipid levels, lipoprotein particle distribution and clearance, and LDLR levels are attenuated in the absence of CEBP $\alpha$. The CEBP $\alpha$ protein is targeted by TRIB1 for degradation, and we previously reported that Trib $1^{\text {shep }}$ mice have increased hepatic CEBP $\alpha$ protein levels (23). To determine if increased CEBP $\alpha$ protein is required for the effect of hepatocyte Trib1 deletion on plasma lipid levels, lipoprotein clearance, and LDLR expression, we generated Trib1 ${ }^{f / f l} \mathrm{Cebpa}^{f / f l}$ mice and injected them with AAV8-TBG-Cre to induce double gene dele-

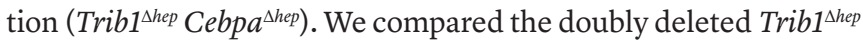
$C e b p a^{\Delta h e p}$ mice with singly deleted Trib1 ${ }^{\Delta h e p}$ and Cebpa $a^{\Delta h e p}$ mice and WT controls also injected with AAV8-TBG-Cre. Liver mRNA levels showed the expected greater than $95 \%$ reductions of Trib1 in Trib1 ${ }^{\text {shep }}$ mice and Cebpa in Cebpa $a^{\Delta h e p}$ mice, as well as a $70 \%$ reduction of Trib1 mRNA in Cebpa $a^{\Delta \text { hep }}$ mice and a 65\% reduction of Cebpa mRNA in Trib1 ${ }^{\Delta h e p}$ mice (Supplemental Figure 5A), attributable to the regulation of both genes by $\mathrm{CEBP} \alpha$ protein. In contrast to the reduction in Cebpa mRNA, the Trib1 ${ }^{\text {shep }}$ mice had a substantial increase in CEBP $\alpha$ protein, particularly the $\mathrm{p} 30$ isoform, whereas the Trib1 $1^{\Delta h e p} C e b p a^{\Delta h e p}$ mice completely lacked CEBP $\alpha$ protein as expected (Supplemental Figure 5B). These findings are consistent with a posttranslational effect of TRIB1 on CEBP $\alpha$ in promoting its degradation.

Deletion of Cebpa in male Trib1 ${ }^{\text {shep }}$ mice eliminated the increase in TC on both chow and WTD (Figure 5A), as well as the increase 
A

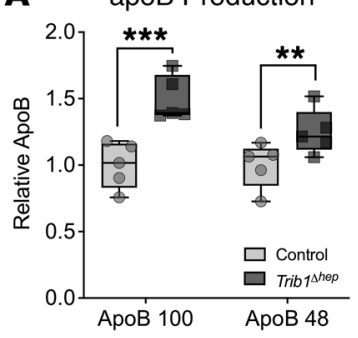

B

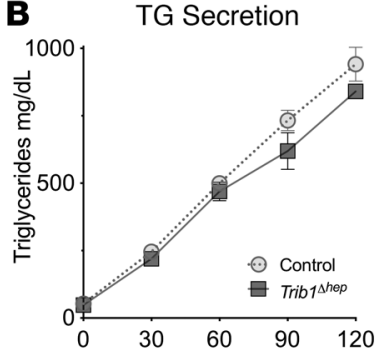

C

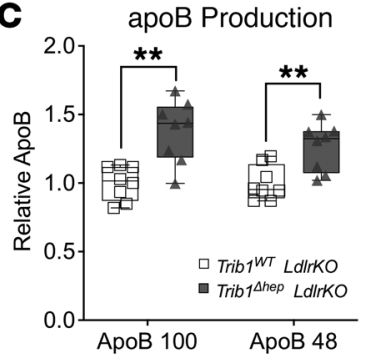

$\mathbf{F}$

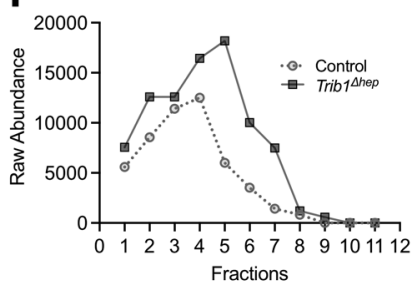

D

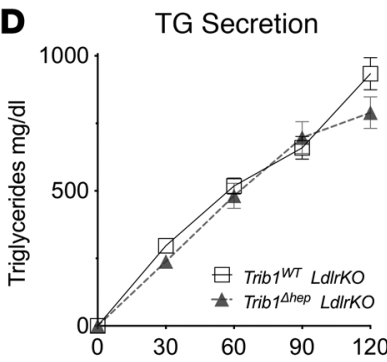

G

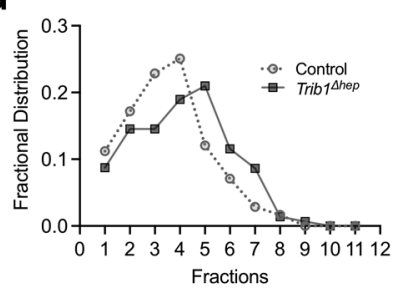

Figure 4. Trib1 ${ }^{\text {thep }}$ mice have increased steady-state plasma lipids due to increased apoB-lipoprotein production. (A and C) Quantification of apoB levels in plasma collected at 1.5 hours after injection with P407 and $200 \mu \mathrm{Ci}$ of ${ }^{35} \mathrm{~S}$ Met/Cys. (B and D) Triglyceride production over time after injection with P407 and $200 \mu \mathrm{Ci}$ of ${ }^{35} \mathrm{~S}$ methionine-cysteine. Experiments were corroborated in $3(\mathbf{A}$ and $\mathbf{B})$ and 2 (C and $\mathbf{D}$ ) independent cohorts. (B and $\left.\mathbf{D}\right)$ Data are expressed as mean \pm SEM for the experimental group. (E) ApoB immunoblot of immunoprecipitated apoB-100 from sucrose density gradient fractionated pooled plasma, 2 hours after injection with P407 and $200 \mu \mathrm{Ci}$ of ${ }^{35} \mathrm{~S}$ Met/Cys. (F) Raw apoB-100 levels and (G) fractional distribution of apoB-100 across the gradient in E. (A and $\mathbf{C}$ ) Box plots indicate median and 25th and 75th percentiles, with whiskers extending to minimum and maximum values. Symbols indicate individual values. Significance was determined by multiple tests with Welch correction, and Benjamini's multiple correction test ( $\left.{ }^{* *} P \leq 0.01,{ }^{* * *} P<0.001\right)$.

in LDL-C on WTD (Figure 5, B and C). Trib1 ${ }^{\text {shep }}$ Cebpa $a^{\Delta h e p}$ mice had normal LDL-apoB catabolism (Figure 5, D and E) and normal VLDL-apoB catabolism (Supplemental Figure 5, C and D), as well as normal levels of $L d l r$ mRNA (Figure $5 F$ ), and normal LDLR protein (Figure 5G and Supplemental Figure 6). Similar studies in female mice recapitulated these phenotypes in the setting of deletion of Cebpa (Supplemental Figure 7). We also performed apoB production studies in this cohort and demonstrated that Trib1 ${ }^{\text {shep }}$ Cebpa ${ }^{\text {shep }}$ mice normalized the rate of apoB production to WT levels, and had a slight but significant decrease in TG secretion consistent with the role of $\mathrm{CEBP} \alpha$ in regulating de novo lipogenesis (Supplemental Figure 5, E and F). These data establish that CEBP $\alpha$ protein is required for the effect of Trib1 deletion in hepatocytes on both LDL-apoB catabolism and apoB secretion and suggest that the increased CEBP $\alpha$ protein in hepatocytes due to loss of Trib1 leads to reduced LDLR expression, slower LDL catabolism, and increased apoB secretion.

Whole-liver transcriptome analysis reveals that most effects of Trib1 $^{\text {shep }}$ are CEBP $\alpha$-dependent. The abundance of hepatic LDLR protein is tightly regulated at multiple levels to ensure normal cellular function, both at transcriptional and posttranscriptional levels (27). To gain further insight about the molecular mechanism underlying the decreased levels of LDLR in Trib1 $1^{\text {shep }}$ mice, we performed whole transcriptome RNA sequencing in livers from chow-fed control, Trib1 ${ }^{\Delta h e p}, C e b p a^{\Delta h e p}$, and Trib1 $1^{\Delta h e p} C e b p a^{\Delta h e p}$ mice. Volcano plot summaries of changes in gene expression between all groups can be found in Supplemental Figure 8, A-E and Supplemental Figure 9, A and B. A total of 14,937 transcripts were differentially expressed between all groups, excluding transcripts with missing values. Out of these, 7458 were upregulated (a positive fold-change relative to WT [ $\left.\log _{2} \mathrm{FC}\right]$ ), and 7479 were downregulat- ed (negative $\log _{2} \mathrm{FC}$ ) in Trib1 $1^{\text {shep }}$ mice compared with control mice. Nine hundred sixteen transcripts (464 up, 452 down) were altered by more than 1.5 -fold between Trib1 ${ }^{\text {shep }}$ and control mice (absolute $\log _{2}$ FC $>0.6$, adjusted $P<0.00001$; Supplemental Figure 8A). Of the 916 differentially expressed transcripts, only 75 met the same adjusted $P$ value cut-off $(P<0.00001)$ in Trib $1^{\text {shep }} C e b p a^{\Delta \text { hep }}$ mouse liver mRNA, indicating that the vast majority (91.8\%) of the significant gene expression changes in Trib1 ${ }^{\text {shep }}$ mouse liver were attributable to dysregulation of CEBP $\alpha$ levels. Trib1 ${ }^{\text {shep }}$ Cebpa $a^{\text {\hep }}$ mouse livers were much more similar to control mice (Supplemental Figure 8B) and the comparison between Cebpa ${ }^{f / f l}$ and Trib $1^{f / f l}$ Cebpa $a^{f / f l}$ showed the fewest changes (Supplemental Figure 8D), consistent with the critical role of elevated CEBP $\alpha$ protein in mediating the effect of Trib1 deletion in liver on gene expression. Of note, the ER stress marker pEIF2a was not elevated in Trib1 $1^{\text {shep }}$ Cebpa ${ }^{\text {shep }}$ mouse livers (Supplemental Figure 4E), indicating that the induction of ER stress by Trib1 deletion is also CEBP $\alpha$ dependent.

Pathway analysis suggests that increased ATF3 contributes to the reduction in LDLR in Trib1 ${ }^{\text {shep }}$ mouse liver. We mined the RNAseq data for transcriptional regulators upstream of $L d l r$ markedly altered by Trib1 deletion that were dependent on CEBP $\alpha$. We noted only modest changes in the expression of the genes encoding the SREBP-1 and -2 proteins (28) and these were not dependent on $\mathrm{CEBP} \alpha$ (Figure 6A, Supplemental Figure 10, and Supplemental Figure 11). Srebf1 mRNA levels were unchanged when comparing all groups (Supplemental Figure 10A), and SREBP1 protein levels were modestly increased in Trib1 $1^{\text {shep }}$, which is the opposite directionality expected for the observed effect on LDLR (Supplemental Figure 10B). Srebf 2 mRNA were decreased in Trib1 ${ }^{\text {shep }}$ as well as in Trib1 ${ }^{\text {Lhep }}$ Cebpa $a^{\text {shep }}$ double mutant mice (Supplemental Figure 10, C and D). However, levels of mature SREBP2 did not differ markedly between 
A

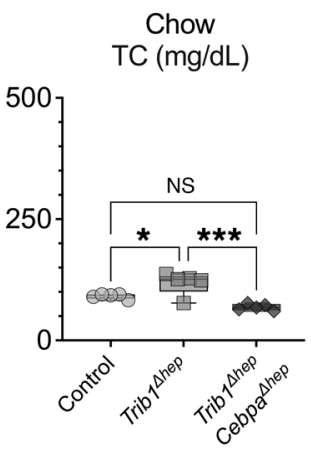

E

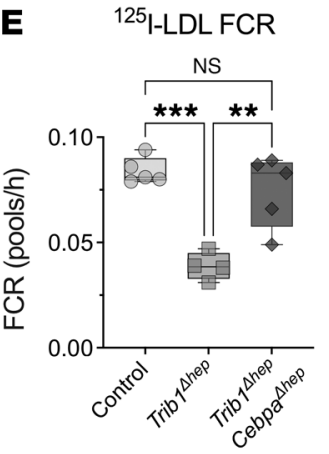

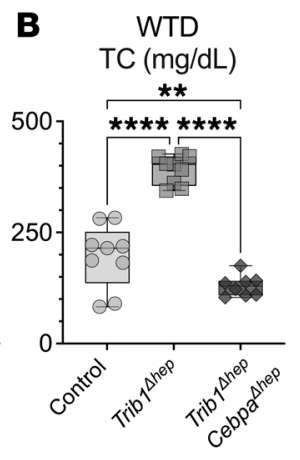

C

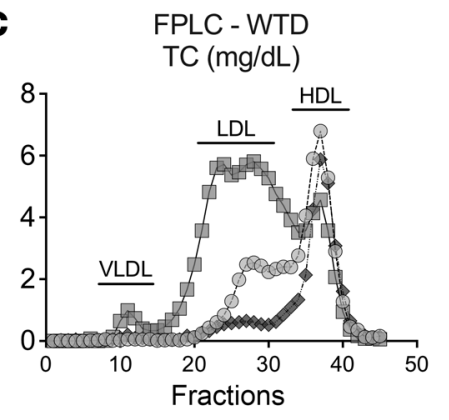

FPLC - WTD

TC $(\mathrm{mg} / \mathrm{dL})$

F LdIr mRNA

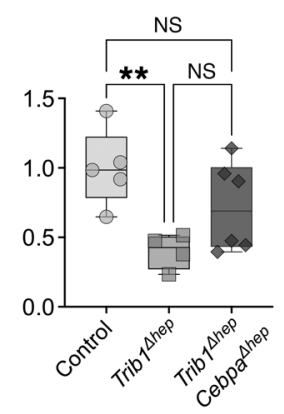

D

${ }^{125}$-LDL clearance

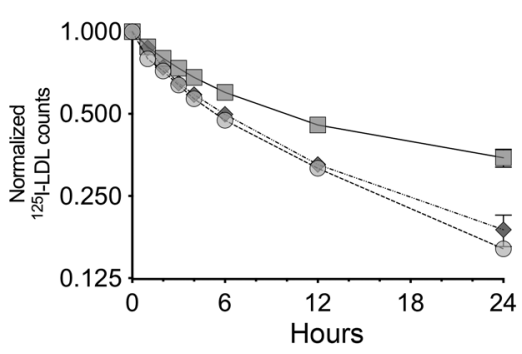

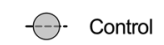
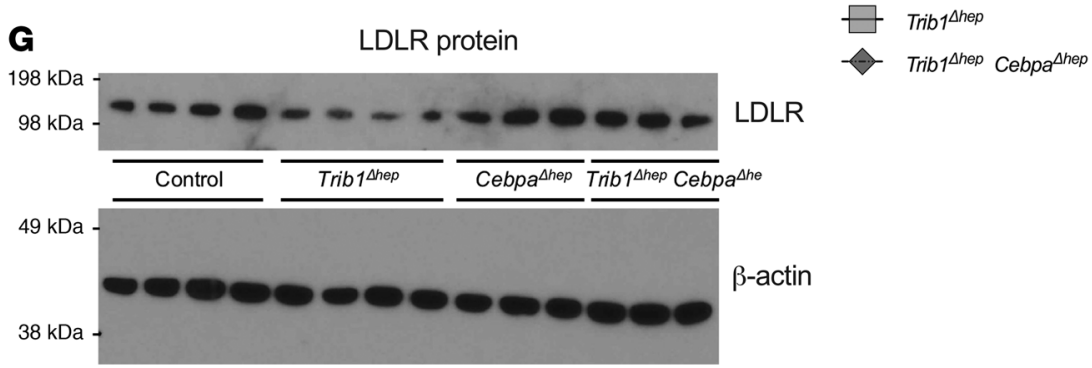

Figure 5. The effects of Trib1 ${ }^{\text {thep }}$ on plasma lipid levels, lipoprotein particle distribution and clearance, and LDLR levels are attenuated in the absence of CEBPa. (A) Plasma levels of total cholesterol in chow-fed male mice 4 weeks after AAV injection $(n=5-6)$ and (B) WTD-fed mice 6 weeks after AAV injection $(n=8-9)$. Results were confirmed in 3 independent cohorts in chow-fed mice and 1 cohort in WTD-fed mice. (C) Total cholesterol distribution in FPLC-fractionated pooled plasma from WTD-fed mice in A. FPLC results were confirmed in 2 independent cohorts in chow-fed mice and 1 cohort in WTD-fed mice. ( $D$ and E) 125I-LDL clearance and FCR in chow-fed male mice 8 weeks after AAV injection $(n=5)$. Results were confirmed in an independent cohort in chow-fed mice and WTD-fed mice. (F) Hepatic transcript levels of Ldlr in mice 8 weeks after AAV injection $(n=5)$, replicated in 3 independent cohorts. (G) Representative immunoblot of hepatic LDLR and $\beta$-actin, which was replicated in 3 independent cohorts. (A, B, E, and F) Box plots indicate median and 25 th and 75 th percentiles, with whiskers extending to minimum and maximum values. Symbols indicate individual values. (D) Data are expressed as mean \pm SEM for the experimental group. Significance was determined by 1-way ANOVA with Tukey's multiple comparison test $\left({ }^{*} P \leq 0.05\right.$, $\left.{ }^{* *} P \leq 0.01,{ }^{* * *} P \leq 0.001,{ }^{* * *} P \leq 0.0001\right)$.

the Trib1 $1^{\text {shep }}$ and control mice. Interestingly, the Trib $1^{\text {shep }} C e b p a^{\Delta \text { she }}$ livers had almost complete loss of mature SREBP2, which may account for the fact that their plasma cholesterol levels are lower than controls. These results suggest that the canonical SREBP pathway has a relatively minor impact on LDLR in hepatocytes lacking TRIB1. In contrast, the activating transcription factor 3 (Atf3) was one of the most highly upregulated genes in Trib1 ${ }^{\text {she }}$ liver (Supplemental Table 1 and Supplemental Figure 9A, 49.3-fold increase, adjusted $P=6.33$ $\times 10^{-46}$ ) and this was dependent on CEBP $\alpha$, as Atf3 expression did not differ between control and Trib1 $1^{\text {shep }}$ Cebpa $a^{\text {shep }}$ livers (Supplemental Table 1 and Supplemental Figure 10B, 1.5-fold increase, adjusted $P=0.57)$. We confirmed these results by qPCR, finding that relative to control, Atf3 mRNA was increased more than 25-fold in Trib $1^{\text {shep }}$ liver but not in Trib1 ${ }^{\text {shep }}$ Cebpa $a^{\text {shep }}$ liver (Figure 6B), which was also confirmed by Western blot (Figure 6C), indicating that increased CEBPa is required for the upregulation of Atf 3 in Trib $1^{\text {hep }}$ mice. Since we had previously performed ChIP-seq for CEBPa in mouse liver, we performed motif analysis on these data and found highly significant enrichment of ATF family binding motifs (including ATF3 and its upstream regulator ATF4) in DNA sequences in chromatin precipitated by antibodies to CEBP $\alpha$ (Figure 6D). Other factors with enriched binding motifs included CEBP $\alpha$ (as expected), NFIL3, CHOP, ATF1, and ATF7 (Supplemental Table 2).
Using pathway analysis, we identified 54 ATF3 downstream target genes that were differentially expressed in the Trib1 ${ }^{\text {shep }}$ mouse liver, and found that these were similarly CEBP $\alpha$ dependent (Supplemental Figure 12, A and B). Pathway analysis of ATF3 downstream genes in other data sets is shown in Supplemental Figure 12, C-E.

Partial silencing of ATF3 in Trib1 ${ }^{\text {shep }}$ mice attenuates the lipid and LDLR phenotypes. To test the hypothesis that preventing ATF3 upregulation in Trib1 $1^{\text {shep }}$ mice would impact LDLR protein and plasma cholesterol levels, we acquired an siRNA directed against mouse Atf 3 and a nontargeting control siRNA (directed against firefly luciferase). We injected Trib1 $1^{\text {shep }}$ mice with siRNAs to Atf3 or luciferase and control mice with siRNA to luciferase. Trib1 $1^{\text {she }}$ mice injected with the luciferase siRNA displayed the expected increase in Atf 3 mRNA whereas in those injected with the Atf 3 siRNA the increase was not significant (Supplemental Figure 13A). More strikingly, the protein abundance of ATF3 was markedly increased in the Trib1 $1^{\text {she }}$ mice receiving the control siRNA, but in Trib1 $1^{\text {she }}$ mice receiving the Atf 3 siRNA this increase in ATF3 was attenuated to levels comparable to control mice (Figure 7A). The decrease in ATF3 protein in the Atf3 siRNA-injected Trib1 $1^{\text {shep }}$ mice was also associated with modestly increased levels of LDLR protein compared with Trib $1^{\text {shep }}$ mice injected with control siRNA (Figure 7B). More importantly, this normalization of ATF3 protein was associated with a significant reduction in the levels of plasma TC throughout 
A

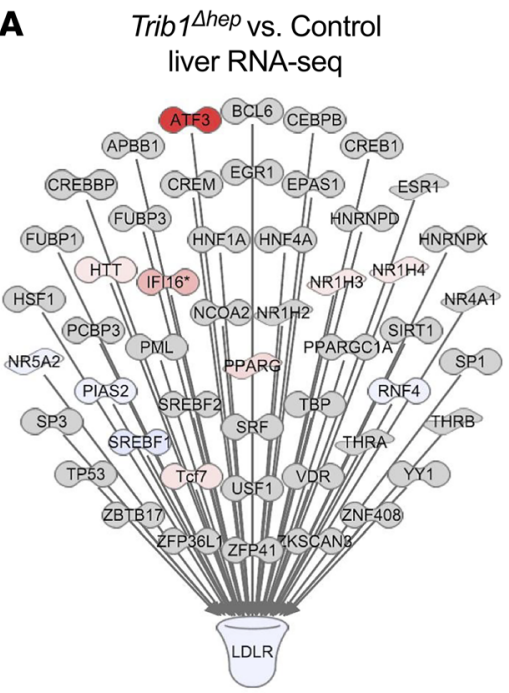

C

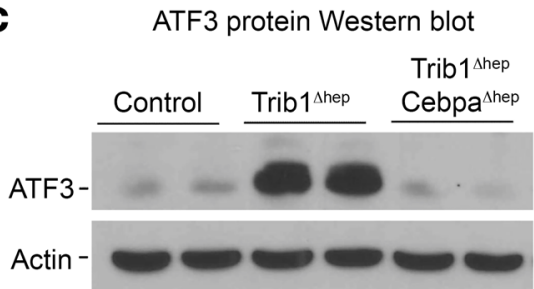

B

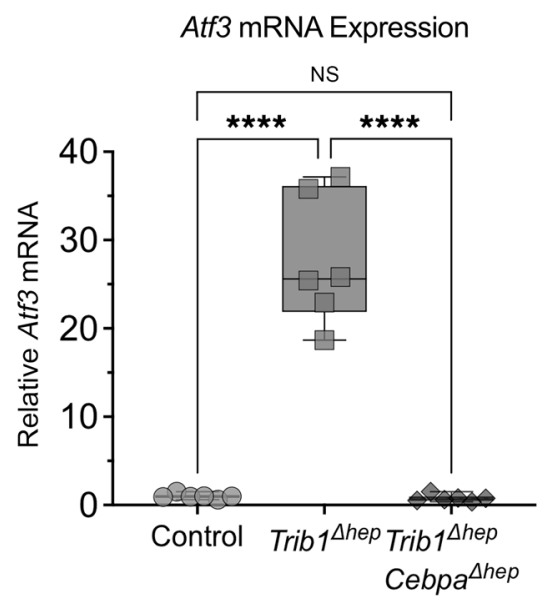

D

\begin{tabular}{|c|c|c|c|}
\hline Motif & TF & Overlap & Adjusted $P$ value \\
\hline CAAC & CEBP & $62.8 \%$ & $1 \mathrm{E}-1016$ \\
\hline ATGATGCAAT & ATF4 & $27.2 \%$ & $1 E-408$ \\
\hline 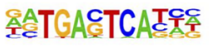 & ATF3 & $14.9 \%$ & $1 E-43$ \\
\hline
\end{tabular}

Figure 6. Pathway analysis suggests that increased ATF3 contributes to the reduction in LDLR in Trib1 ${ }^{\text {thep }}$ mouse liver. (A) Ingenuity pathway analysis (IPA) of all predicted liver-expressed ligand-dependent nuclear receptors, transcription factors, and transcriptional regulators upstream of Ldlr (based on IPA knowledge base) overlaid with color-coded mouse liver RNA-Seq data from the comparison of Trib1 ${ }^{\text {thep }}$ transcript abundance to control. Analysis was performed on total liver RNA from chow-fed mice 4 weeks after AAV8-TBG-Cre injection $(n=6)$. (B) Hepatic transcript levels of ATF3 in chow-fed male mice ( $n=6$ per group) 4 weeks after injection AAV8-TBG-Cre. (C) Immunoblot of hepatic ATF3 and $\beta$-actin, replicated in 3 independent cohorts. (D) Motif enrichment analysis of CEBP $\alpha$ ChIP-seq in Trib1 ${ }^{\text {thep }}$ liver shows significant enrichment of ATF proteins. (B) Box plots indicate median and 25 th and 75 th percentiles, with whiskers extending to minimum and maximum values. Symbols indicate individual values. Data are expressed as mean \pm SEM for the experimental group. Significance was determined by 1-way ANOVA with Tukey's multiple comparison test $\left.{ }^{* * * *} P \leq 0.0001\right)$.

the time course of the experiment (Figure 7, C-E). Other plasma lipid fractions were not markedly affected by the Atf3 siRNA (Supplemental Figure 12, B-K). This result supports the model that ATF3 contributes to the downregulation of LDLR in Trib1 $1^{\text {sep }}$ mice, while allowing that other factors are also likely involved.

\section{Discussion}

Here we demonstrate that deletion of Trib1 in mouse liver increases plasma cholesterol and apoB levels in part through substantial downregulation of the LDLR and reduced clearance of apoB-containing lipoproteins. In addition, it also increases the hepatic production of newly synthesized apoB, which contributes to the increased plasma apoB. Both effects of Trib1 deletion are mediated by an increase in CEBP $\alpha$ and are abolished in the setting in which Cebpa is also deleted in liver. We found that Atf 3 is markedly upregulated by Trib1 deletion in a Cebpa-dependent manner, and that partial silencing of Atf 3 blunted the effects of Trib1 deletion on the LDLR and plasma cholesterol. Thus, we establish a new pathway involving TRIB1/CEBP $\alpha / A T F 3$ that contributes to the regulation of the LDLR and apoB lipoprotein clearance and may help explain the association of genetic variants at the TRIB1 locus with LDL-C and apoB levels in humans.
Previous work has shown that TRIB1 acts as a scaffolding protein that contributes to the destabilization of CEBP $\alpha$ protein by promoting ubiquitin conjugation of CEBP $\alpha$ by the COP1/RFWD2 E3 ubiquitin ligase (15-18). As a result, Trib1 ${ }^{\text {she }}$ mice have elevated levels of hepatic CEBP $\alpha$. Our previous work has shown that the increased CEBP $\alpha$ drives increased liver expression of the genes in the hepatic de novo lipogenesis pathway, leading to hepatic steatosis associated with increased plasma lipids $(22,23)$. Here, we addressed the mechanisms underlying the plasma lipid phenotype in Trib1 ${ }^{\text {shep }}$ mice and show for the first time that these mice have decreased levels of hepatic Ldlr mRNA and LDLR protein, and that as a result they exhibit markedly impaired clearance of apoB-lipoprotein particles. In formally testing the dependence of this phenotype on LDLR, by comparing homozygous Ldlr-KO mice that were either Trib ${ }^{W T}$ or Trib1 $1^{\text {shep }}$, we found that in addition to the particle clearance phenotype, Trib1 ${ }^{\text {shep }}$ mice have increased hepatic production of apoB particles that is not associated with a change in hepatic TG secretion. Physiologically, this represents a double hit: the Trib $1^{\text {she }}$ livers not only have a reduced capacity to clear apoB particles, but also produce apoB lipoproteins in greater numbers. To elucidate the underlying molecular mechanism by which TRIB1 regulates the LDLR, we performed a 4-arm hypoth- 
A

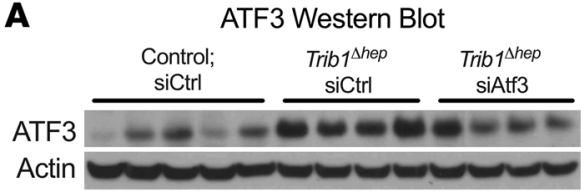

B

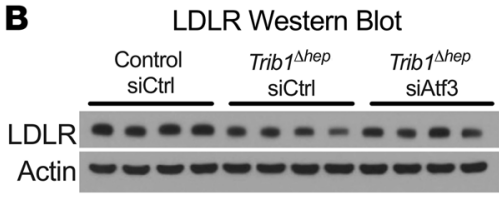

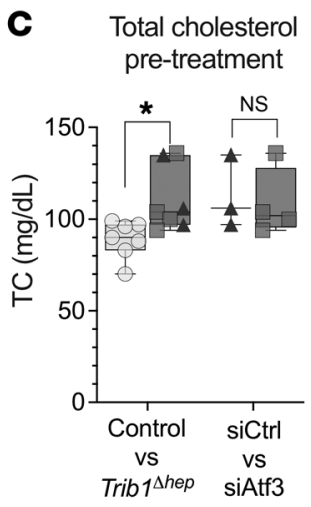

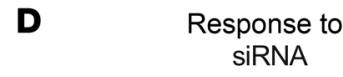
SiRNA

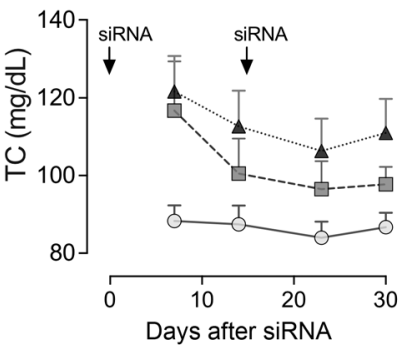

E siRNA response AUC

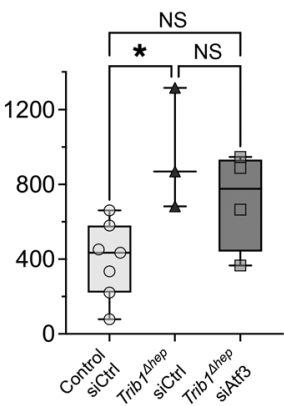

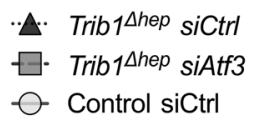

Figure 7. Partial silencing of ATF3 in Trib1 ${ }^{\text {hep }}$ mice attenuates the plasma lipid phenotype. (A) Hepatic ATF3 in chow-fed male mice (Trib1 ${ }^{\mathrm{WT}}$ siCtrl, $n=5$; Trib1 $^{\text {Ahep }}$ siCtrl, $n=4$; Trib1 ${ }^{\Delta \text { hep }} \operatorname{siAtf3,} n=4$ ) at the end of the siRNA knockdown time course. (B) Total cholesterol prior to siRNA treatment ( $n=7$ Control siCtrl [siRNA against luciferase; $n=3$ Trib1 $^{\Delta h e p}$ siCtrl; and $n=4$ Trib1 $^{\Delta \text { hep }}$ siAtf3. Left panel shows all Trib1 ${ }^{\Delta h e p}$ mice, right grouping shows the assignment of the Trib1 ${ }^{\text {thep }}$ mice by eventual siRNA group (triangles, siCtrl; squares, siAtf3). (C) Time course of plasma total cholesterol in siRNA-injected mice. (D) Area under the curve of the siRNA treatment time course in panel E; $y$ axis, arbitrary units. (B) Box plots indicate median and 25th and 75th percentiles, with whiskers extending to minimum and maximum values. Symbols indicate individual values. Data are expressed as mean \pm SEM for the experimental group. Significance in B was determined by multiple unpaired $t$ tests with Welch correction, and Benjamini's multiple correction test $\left({ }^{*} P \leq 0.05\right)$ and in $\mathbf{D}$ by 1 -way ANOVA of individual AUC values (baseline set to 65) by Kruskal-Wallis with Dunn's correction for multiple testing (adjusted ${ }^{*} P \leq 0.05$ ). The siRNA experiment represents a single cohort.

esis-generating RNA-sequencing experiment, profiling hepatic gene expression in control (Trib1 $\left.{ }^{W T}\right)$, Trib1 $^{\Delta h e p}, C e b p a^{\Delta h e p}$, and doubly deleted Trib1 ${ }^{\Delta h e p} C e b p a^{\Delta h e p}$ mice. Pathway analysis comparisons of the control and Trib1 ${ }^{\Delta h e p}$ data sets identified ATF3 as the most markedly dysregulated upstream regulator of LDLR, and importantly, showed that the dysregulation of Atf3 did not occur if CEBP $\alpha$ was deleted along with TRIB1.

ATF3 is a stress-inducible gene that encodes a member of the ATF/CREB family of transcription factors, which share a basic-region leucine zipper (bZip) DNA binding domain (29). ATF3 expression is low in cells at steady state and is induced by a variety of extracellular stimuli, such high concentrations of glucose and palmitate, and ER stress (30). Further, ATF3 has been associated with the modulation of the immune response, atherogenesis, glucose homeostasis, hepatic steatosis, diabetes, and atherosclerosis $(30,31)$. A previous study using human liver Sk-Hep1 cells provided evidence that increased ER stress response or disturbance of mitochondrial function leads to the activation of ATF3, which causes a reduction in LDLR expression (32). They demonstrated that the region of the $L D L R$ proximal promoter region is a putative binding site for ATF3 and confirmed the interaction by chromatin immunoprecipitation in a stress-dependent manner (32). Previous studies of bZip protein-protein interaction networks using electrophoretic mobility shift assay (EMSA), and fluorescence resonance energy transfer (FRET) demonstrated that ATF3 physically interacts with CEBP $\alpha$ (33). A second study using both EMSA and ChIP assays demonstrated that ATF3 directly binds to the human CEBPA promoter (34), and conversely CEBP $\alpha$ has been shown to activate transcription of ATF3 from the ATF3 promoter (35), supporting the notion of mutual regulation of CEBP $\alpha$ and ATF3.
A recent publication by $\mathrm{Xu}$ et al. (36) investigated the effects of hepatocyte ATF3 in the regulation of bile acids and HDL metabolism and atherosclerosis and demonstrated that hepatocyte ATF3 enhances HDL uptake, inhibits intestinal fat and cholesterol absorption, and promotes macrophage reverse cholesterol transport. Consistent with earlier studies (31), they found that the mechanism by which ATF3 regulates these is by inducing scavenger receptor group B type 1 (SR-BI) and repressing cholesterol 12 $\alpha$-hydroxylase (CYP8B1) in the liver through its interaction with p53 and HNF4A. Additionally, they explored the effects of hepatic ATF3 on the LDLR and showed that ATF3 upregulates hepatic expression of LDLR, a direction of effect opposite to the previously published results by Lim et al. (32), on which we based our hypothesis. We speculate that this may be due to the profoundly supraphysiological overexpression of ATF3 (>200-fold over control) in the recent study (36). In addition, in our Trib1 ${ }^{\text {shep }}$ mice, levels of both ATF3 and $\mathrm{CEBP} \alpha$ are elevated; the concomitant elevation of $\mathrm{CEBP} \alpha$ may change the nature of the effect of ATF3 on Ldlr transcription.

Our results are consistent with a model in which the deletion of Trib1 allows CEBP $\alpha$ protein to escape targeted proteolysis (via COP1/ RFWD2), resulting in increased CEBP $\alpha$ protein. The increased CEBP $\alpha$ protein directly interacts with the Atf3 promoter and leads to its transcriptional upregulation. The ATF3 protein (which $\mathrm{CEBP} \alpha$ also binds directly) binds the $L d l r$ promoter and represses its transcription, thereby resulting in the downregulation of LDLR mRNA and consequently of LDLR protein. The functional importance of the ATF3 upregulation in Trib $1^{\text {shep }}$ mice is further supported by the finding that there is a highly significant enrichment of ATF family DNA binding motifs in ChIP-seq peaks generated by anti-CEBP $\alpha$ pull-down of Trib1 ${ }^{\text {shep }}$ mouse livers. 
In summary, our experiments show that deletion of hepatic Trib1 increases plasma LDL-C levels by resulting in downregulation of the expression of the LDLR, leading to reduced LDL-apoB clearance, and that this effect of TRIB1 is dependent on CEBP $\alpha$. We also identified ATF3 as at least one mediator of the effects of TRIB1 and CEBP $\alpha$ regulation of LDLR expression. This study provides new functional insight into the strong genetic association between TRIB1 and LDL-C levels in humans.

\section{Methods}

Animals. Trib1 genetically engineered mice C57BL/6-Trib1 $\mathrm{t}^{\mathrm{m} 1.1 \mathrm{mrl}}$ were provided by Merck and were produced for Merck under contract by Taconic Biosciences (37). Previously reported (38) mice harboring a conditional allele of Cebpa $\left(\mathrm{Cebpa}^{\mathrm{tm} 1 \mathrm{Dgt}} / \mathrm{J}\right)$ were obtained from The Jackson Laboratory (stock number 006447). C57BL/6J WT mice were obtained by the Jackson Laboratory (000664). Mice were maintained in a monitored small animal facility at the University of Pennsylvania under Institutional Animal Care and Use Committee-approved protocols and were fed ad libitum with a either standard chow diet or (where explicitly noted in the text) WTD containing $40 \% \mathrm{kCal}$ of fat, $43 \% \mathrm{kCal}$ carbohydrate, and $17 \%$ $\mathrm{kCal}$ of protein from OpenSource Diets (D12079B, Research Diets) for the indicated periods of time. For experiments in WTD, mice were injected with AAV8-TBG-Cre, plasma lipids were monitored for 5 weeks in chow fed diet, and WTD was introduced 5 weeks after deletion. All mice were provided access to water ad libitum and were maintained with a 12-hour on/12-hour off light cycle with lights off from 7:00 p.m. to 7:00 a.m. daily. For all studies, male mice approximately 10 to 12 weeks of age were used; for some of the studies, female mice of similar age were also included. Five to ten mice per group were used for all studies.

Adeno-associated 8 (AAV-8) viral vector preparation. AAV serotype 8 vectors containing an empty expression cassette (AAV8-Null) or encoding Cre recombinase (AAV8-TBG-Cre) were generated by the University of Pennsylvania Vector Core (Philadelphia, Pennsylvania, USA). The transgene in these vectors is selectively expressed in hepatocytes from the thyroxine-binding globulin (TBG/Serpina7) promoter (39). For all experiments utilizing AAV8-TBG-Cre, animals were injected with AAV vectors at a dose of $1.5 \times 10^{11}$ genome copies per mouse (6) via i.p. injection and examined at the stated time points. Control mice refer to either Trib1 $1^{f / f l}$ mice receiving AAV8-TBG-Null (empty vector) or Trib1 ${ }^{W T}$ mice receiving AAV8-TBG-Cre.

Atf3 and luciferase control siRNA injections. Atf3 and luciferase control siRNA were provided by Alnylam. Control and Trib $1^{\text {shep }}$ mice were injected with either a control siRNA (directed against luciferase, not expressed in the experimental mice) or siRNA targeting Atf 3 at a dose of $10 \mathrm{mg} / \mathrm{kg}$ and examined at the stated time points.

Plasma lipid phenotypic characterization. All blood samples from mice were collected by retro-orbital bleeding from mice anesthetized with isoflurane using EDTA-coated glass tubes under approved protocols. Blood was centrifuged at 7,700 x g (average radius) for 7 minutes at $4^{\circ} \mathrm{C}$ and plasma was obtained. Plasma TC, HDL-C, and TG levels were measured using an Axcel clinical autoanalyzer (Alfa Wassermann Diagnostic Technologies). Non-HDL-C was calculated by the difference between the TC and HDL-C measurements. All measurements were made from 4 hour-fasted plasma samples unless stated otherwise. In addition, plasma samples were pooled by experimental group after collection and $150 \mu \mathrm{L}$ plasma was separated by FPLC on a Superose 6 gel-filtration column (GE Healthcare Life Sciences) into $0.5 \mathrm{~mL}$ fractions. TC and TG were measured from FPLC-separated fractions by colorimetric assay (Infinity TG/TC, Thermo Fisher Scientific).

$L D L-a p o B$ and VLDLR- ароB kinetic experiments. LDL was isolated from human plasma by ultracentrifugation. Purified LDL was iodinated with ${ }^{125}$ I directly using the iodine monochloride method (40). The final iodinated product was dialyzed against PBS before measurement of protein concentration by BCA assay and ${ }^{125}$ I counts by gamma counting. For ${ }^{125} \mathrm{I}-\mathrm{LDL}-\mathrm{apoB}$ clearance studies, iodinated LDL-apoB-specific activity was approximately 50 to 100 counts/ng protein. Mice were administered by intravenous tail vein injection with approximately 2 million counts ${ }^{125}$ I-LDL in $100 \mu \mathrm{L}$ plasma. Mice were bled 2 minutes, 1, 2, 3, 4, 6, 12, and 24 hours after radioisotope administration and sacrificed at 24 hours. ${ }^{125} \mathrm{I}$ activity at each time point was measured from $5 \mu \mathrm{L}$ of each plasma sample by counting on a Packard Cobra II Auto-Gamma counter. The relative activity at each time point was determined as the fraction of activity at 2 minutes for each mouse respectively. For ${ }^{125}$ I-VLDL-apoB clearance studies, iodinated VLDL-apoB-specific activity was approximately 50 to 100 counts/ng protein. Mice were administered by intravenous tail vein injection with approximately 5 million counts ${ }^{125} \mathrm{I}-\mathrm{VLDL}$ in $100 \mu \mathrm{L}$ plasma diluted in PBS. Mice were bled 1 minute, 5 minutes, 15 minutes, 30 minutes, 1 hour, 3 hours, 6 hours, and 24 hours after radioisotope administration and sacrificed at 24 hours. ${ }^{125}$ I activity at each time point was measured from $5 \mu \mathrm{L}$ of each plasma sample by counting on a Packard Cobra II Auto-Gamma counter. To get VLDL-apoB-specific counts, plasma was subjected to isopropanol precipitation and the isopropanol precipitate was measured for counts on the gamma counter. The relative activity at each time point was determined as the fraction of activity at 1 minute for each mouse respectively.

$T G$ and $a p o B$ secretion experiments. Mice were fasted for 4 hours and then injected intravenously with $0.67 \mathrm{mg} / \mathrm{g}$ of body weight pluronic F127 Nf Prill Poloxamer 407 (P407) (BASF, material 30085239) and 300 to $400 \mu \mathrm{Ci}$ Easytag EXPRES ${ }^{35}$ S protein labeling mix ( $\left.{ }^{35} \mathrm{SMet} / \mathrm{Cys}\right)$ (Perkin Elmer, NEG772). Blood samples were taken immediately prior to the $\mathrm{P} 407 /{ }^{35} \mathrm{~S}$ Met/Cys injection and at 30, 60, 90, and 120 minutes after injection. The blood was placed on ice and spun for 7 minutes at $10,000 \mathrm{~g}$ and plasma transferred within 15 minutes of collection. Plasma TG concentration was measured at all time points by colorimetric assay (Infinity TG, Thermo Fisher Scientific).

Newly synthesized and secreted total plasma apoB100 was measured by the incorporation of ${ }^{35} \mathrm{~S}-\mathrm{Met} / \mathrm{Cys}$ into apoB-100 that was secreted into the systemic circulation during the same 2-hour period. Total plasma was boiled in Laemmli sample buffer and separated on a $3 \%$ to $8 \%$ Tris Acetate gel (Novex); the gel was dried onto filter paper under vacuum, and the labeled proteins visualized by autoradiography. The apoB-100 bands were cut and analyzed for $\left.{ }^{[35} \mathrm{S}\right]$ activity by liquid scintillation counting. To mitigate differences in total labeled hepatic protein synthesis, counts were adjusted based on the incorporation of ${ }^{35} \mathrm{~S}$-methionine into all proteins in plasma trichloroacetic acid (TCA) precipitated protein counts. To determine TCA precipitated protein counts, $5 \mu \mathrm{L}$ plasma from the sample was blotted onto a $1 \times 1 \mathrm{~cm}$ square of filter paper. Plasma proteins were precipitated by incubating the filter paper in $20 \%$ TCA 
on ice followed by $10 \%$ TCA heated to $100^{\circ} \mathrm{C}$. The filter paper was subsequently rinsed with $100 \%$ ethanol, dried, and ${ }^{35}$ S activity calculated by liquid scintillation counter.

Sucrose gradient ultracentrifugation experiment. Sucrose gradient ultracentrifugation of apoB-containing lipoproteins was conducted as described (41). All solutions contained $0.1 \mathrm{mM}$ leupeptin, $1 \mu \mathrm{M}$ pepstatin $\mathrm{A}$, $0.86 \mathrm{mM}$ phenylmethylsulfonyl fluoride, $100 \mathrm{U} / \mathrm{mL}$ aprotinin, $5 \mu \mathrm{M}$ ALLN, $5 \mu \mathrm{M}$ EDTA, $150 \mathrm{mM} \mathrm{NaCl}$, and $50 \mathrm{mM}$ phosphate-buffered saline, $\mathrm{pH}$ 7.4. The sample layer was prepared by diluting $200 \mu \mathrm{L}$ of pooled plasma from the $120 \mathrm{~min}$ utes postinjection time point from mice in the apoB secretion studies in $2.3 \mathrm{~mL}$ PBS and adding $2.5 \mathrm{~mL}$ of $25 \%$ sucrose in PBS. The sucrose gradient was formed by layering from the bottom of the tube: $2 \mathrm{~mL}$ of $47 \%$ sucrose, $2 \mathrm{~mL}$ of $25 \%$ sucrose, $5 \mathrm{~mL}$ of sample in $12.5 \%$ sucrose, and $3 \mathrm{~mL}$ of phosphate-buffered saline. The gradients were spun at 151,000 $\mathrm{xg}$ (average radius) in a Beckman SW41 rotor for 65 hours at $12^{\circ} \mathrm{C}$, and then 12 fractions were removed from the top to the bottom and subjected to immunoprecipitation for apoB, separated by gel electrophoresis, and the apoB protein detected by Western blot.

Western blot analysis. Protein extracts were prepared from approximately $100 \mathrm{mg}$ of liver tissue homogenized in lysis buffer (25 mM Tris-HCL, $10 \mathrm{mM} \mathrm{Na} \mathrm{P}_{2} \mathrm{O}_{7}$, $1 \%$ NP40, $10 \mathrm{mM} \mathrm{NaF}, 1 \mathrm{mM}$ EGTA, $1 \mathrm{mM}$ EDTA, $1 \mathrm{mM}$ PMSF, $5 \mu \mathrm{g} / \mu \mathrm{L}$ leupeptin, $5 \mu \mathrm{g} / \mu \mathrm{L} 10$ $\mathrm{nM}$ okadaic acid, protease inhibitor tablet [Roche], and phospho tablet [Roche]). Samples were homogenized, shaken for 1 hour at $4^{\circ} \mathrm{C}$ and centrifuged at $12,000 \mathrm{~g}$ for 10 minutes. Protein was separated by SDS-PAGE using the NuPAGE system (Invitrogen). ApoB antibody was acquired from Abcam (ab31992), LDLR antibody was acquired from Abcam (ab52818), monoclonal anti B-Actin antibody-clone AC-15 was acquired from Sigma-Aldrich (A5441), CEBP $\alpha$ antibody was obtained from Cell Signaling (D56F10), and phospho-eIF2a antibody was obtained from ENZO (BML-SA405). For ATF3, SREBP1 and SREBP2, a nuclear enrichment protocol, was used. Protein extracts were prepared from approximately 100 $\mathrm{mg}$ of liver tissue homogenized in lysis buffer (50 mM HEPES, 10 $\mathrm{mM} \mathrm{Na} \mathrm{P}_{2} \mathrm{O}_{7}, 1 \% \mathrm{NP} 40,300 \mathrm{mM} \mathrm{NaCl}, 0.2 \mathrm{mM}$ EGTA, $10 \mathrm{mM}$ EDTA, $1 \mathrm{mM} \mathrm{MgCl}, 1 \mathrm{mM} \mathrm{CaCl}, 1 \mathrm{mM}$ PMSF, $5 \mu \mathrm{g} / \mu \mathrm{L}$ leupeptin, $5 \mu \mathrm{g} / \mu \mathrm{L} 10 \mathrm{nM}$ okadaic acid, protease inhibitor tablet [Roche], and $20 \%$ glycerol). Samples were homogenized, shaken for an hour at $4^{\circ} \mathrm{C}$, centrifuged at $15,000 \mathrm{~g}$ for 30 minutes and then the supernatant was sonicated. Protein was separated by SDS-PAGE using the NuPAGE system (Invitrogen). ATF3 antibody (NBP1-85816) and SREBP2 antibody (NB100-74543) were obtained from Novus Biologicals. SREBP1 antibody (2A4) (sc-13551) was from Santa Cruz Biotechnology. ECL HRP-conjugated secondary antibodies were from GE Healthcare Life Sciences and the Crescendo ECL reagent (Millipore) was used for protein development.

RNA isolation and quantitative RT-PCR. RNA was collected from approximately $100 \mathrm{mg}$ flash-frozen liver tissue using the mirVana Kit (Invitrogen) following the manufacturer's protocol. cDNA was produced from $1 \mu \mathrm{g}$ total RNA with the High-Capacity cDNA Reverse Transcription Kit (Applied Biosystems) following the manufacturer's instructions. Real-time PCR analysis was performed on a QuantStudio RT-PCR machine. The relative quantity of each mRNA was calculated using the $\Delta \Delta \mathrm{Ct}$ method and normalized to the combined mean Ct of Mrlp19, Ywhaz, and Ipo8.
The TaqMan Gene Expression probes (Invitrogen) used were as follows: Trib1, Mm00454875_m1; Cebpa, Mm00514283_s1; Ldlr, Mm01177349_m1; Atf3, Mm00476033_m1; Srebf1, Mm00550338_ m1; Srebf2, Mm01306292_m1, Mrlp19, Mm00452754_m1; Ywhaz, Mm03950126_s1; and Ipo8, Mm01255158_m1.

Statistics. All data are reported as the mean \pm SEM. Statistical comparisons between 2 groups were performed using a 2-tailed Student's $t$ test or 1-way ANOVA as appropriate and when assumptions of distribution of the data were valid. Comparisons among 3 experimental groups were performed using 2-way ANOVA. Statistical significance was defined as $P$ less than 0.05 .

Gene expression profiling (RNA-Seq) and Ingenuity Pathway Analysis. RNA was collected from approximately $100 \mathrm{mg}$ flash-frozen liver tissue using the mirVana Kit (Invitrogen) following the manufacturer's protocol. Total RNA was analyzed using the Agilent RNA 600 Nano Kit following the manufacturer's protocol. A whole-transcriptome, high-throughput library was prepared using Illumina truSeq stranded mRNA kit. Sequencing was performed on a HiSeq4000 (100 bp, single end reads), to get approximately 312 million reads. RNA-Seq and data analysis were performed by the Penn Functional Genomics Core. Raw sequence files (fastq) for 27 samples were mapped to the transcriptome using Salmon (42) against the mouse transcripts described in genecode (version M22, built on the mouse genome GRCm38.p6; ref. 43). Transcript counts were summarized to the gene level using tximport (44) and normalized and tested for differential expression using DESeq2 (45). Differential expression was determined using a FDR cutoff of $10 \%$.

Transparency disclosure. The lipoprotein clearance data from the control and Trib $1^{\text {shep }}$ groups shown in Figure 2, A and B are repeated in Figure 5C. Similarly, the control data in Figure 2, A and $\mathrm{B}$ are also repeated in Figure 3, A and B. The control data in Figure 2, $\mathrm{E}$ and $\mathrm{F}$ are the same as those shown in Figure 3, C and D. These experiments were performed together but are separated in the text for narrative clarity.

Study approval. Animal experiments were reviewed and approved by the Institutional Animal Care and Use Committee of the University of Pennsylvania, Philadelphia, Pennsylvania, USA. The data discussed in this publication have been deposited in NCBI's Gene Expression Omnibus (46) and are accessible through GEO Series accession number GSE158815.

\section{Author contributions}

DJR conceptualized and acquired funding for the study. RCB, $\mathrm{NJH}$, and DJR designed the original study. KQF led all and conducted most of the experimental work, with selective participation by CV (lipoprotein clearance assays, selected Western blots) and DMC (apoB-lipoprotein production and TG secretion experiment, selected Western blots). All authors analyzed data. JSM performed kinetics modeling. JWT and NJH performed bioinformatics. KQF, $\mathrm{NJH}$, and DJR wrote the manuscript.

\section{Acknowledgments}

We thank Alnylam Pharmaceuticals for the gift of the siRNAs used in this study. The authors wish to thank Aisha Wilson, Maosen Sun, Edwige Edouard, Debra Cromley, Kevin Trindade, Linda Morell, Amrith Rodrigues, Mikhaila Smith, and Andrea Berrido for expert technical assistance, Dawn Marchadier for project man- 
agement, and Linda Carmichael and Stephanie DerOhannessian for additional administrative support. We gratefully acknowledge Jeff Billheimer and Warren Pear for helpful discussions regarding our data, and past and present members of the Rader lab for their insights and suggestions. This work was supported by National Institutes of Health grants R01HL134853 (to NJH and DJR) and F31HL143857 (to KQF), and by American Heart Award grants 18POST34080184 (to CV) and 16SDG31180039 (to RCB).
Address correspondence to: Nicholas J. Hand or Daniel J. Rader, University of Pennsylvania, 11-135 Smilow Center for Translational Research, 3400 Civic Center Boulevard, Philadelphia, Pennsylvania, 19104-5158, USA. Email: njhand@pennmedicine.upenn. edu (NJH); rader@pennmedicine.upenn.edu (DJR).

RCB's present address is: Department of Medicine, Columbia University Medical Center, New York, New York, USA.
1. Kathiresan S, et al. Six new loci associated with blood low-density lipoprotein cholesterol, high-density lipoprotein cholesterol or triglycerides in humans. Nat Genet. 2008;40(2):189-197.

2. Willer CJ, et al. Newly identified loci that influence lipid concentrations and risk of coronary artery disease. Nat Genet. 2008;40(2):161-169.

3. Teslovich TM, et al. Biological, clinical and population relevance of 95 loci for blood lipids. Nature. 2010;466(7307):707-713.

4. Aung LHH, et al. Association of the TRIB1 tribbles homolog 1 gene rs17321515 A $>\mathrm{G}$ polymorphism and serum lipid levels in the Mulao and Han populations. Lipids Health Dis. 2011;10(1):230.

5. Chambers JC, et al. Genome-wide association study identifies loci influencing concentrations of liver enzymes in plasma. Nat Genet. 2011;43(11):1131-1138.

6. Dastani Z, et al. Novel loci for adiponectin levels and their influence on type 2 diabetes and metabolic traits: a multi-ethnic meta-analysis of 45,891 individuals. PLoS Genet. 2012;8(3):e1002607.

7. Klarin D, et al. Genetics of blood lipids among 300,000 multi-ethnic participants of the Million Veteran Program. Nat Genet. 2018;50(11):1514-1523.

8. Liu Q, et al. TRIB1 rs17321515 and rs2954029 gene polymorphisms increase the risk of non-alcoholic fatty liver disease in Chinese Han population. Lipids Health Dis. 2019;18(1):61.

9. Juan Mata SCAEPR. Tribbles coordinates mitosis and morphogenesis in drosophila by regulating string/CDC25 proteolysis. Cell. 2000;101:511-522.

10. Seher TC, Leptin M. Tribbles, a cell-cycle brake that coordinates proliferation and morphogenesis during Drosophila gastrulation. Curr Biol. 2000;10(11):623-629.

11. Rg Großhans J, Wieschaus E. A genetic link between morphogenesis and cell division during formation of the ventral furrow in drosophila. Cell. 2000;101:523-531.

12. Yokoyama T, Nakamura T. Tribbles in disease: signaling pathways important for cellular function and neoplastic transformation. Cancer Sci. 2011;102(6):1115-1122.

13. Dobens LL, Bouyain S. Developmental roles of tribbles protein family members. Dev Dyn. 2012;241(8):1239-1248.

14. Eyers PA, et al. Tribbles in the 21st century: the evolving roles of tribbles pseudokinases in biology and disease. Trends Cell Biol. 2017;27(4):284-298.

15. Dedhia PH, et al. Differential ability of Tribbles family members to promote degradation of $\mathrm{C}$ /
EBPalpha and induce acute myelogenous leukemia. Blood. 2010;116(8):1321-1328.

16. Murphy JM, et al. Molecular mechanism of CCAAT-enhancer binding protein recruitment by the TRIB1 pseudokinase. Structure. 2015;23(11):2111-2121.

17. Nakamura T. The role of Trib1 in myeloid leukaemogenesis and differentiation. Biochem Soc Trans. 2015;43(5):1104-1107.

18. Satoh T, et al. Critical role of Trib1 in differentiation of tissue-resident M2-like macrophages. Nature. 2013;495(7442):524-528.

19. Makishima S, et al. Sin3A-associated protein, 18 $\mathrm{kDa}$, a novel binding partner of TRIB1, regulates MTTP expression. JLipid Res. 2015;56(6):1145-1152.

20. Soubeyrand $S$, et al. TRIB1 is a positive regulator of hepatocyte nuclear factor 4-alpha. Sci Rep. 2017;7(1):1-12.

21. Ishizuka Y, et al. TRIB1 downregulates hepatic lipogenesis and glycogenesis via multiple molecular interactions. JMol Endocrinol. 2014;52(2):145-158.

22. Burkhardt R, et al. Trib1 is a lipid- and myocardia infarction-associated gene that regulates hepatic lipogenesis and VLDL production in mice. J Clin Invest. 2010;120(12):4410-4414.

23. Bauer RC, et al. Tribbles-1 regulates hepatic lipogenesis through posttranscriptional regulation of C/EBP $\alpha$. J Clin Invest. 2015;125(10):3809-3818.

24. Chemello K, et al. Lipoprotein metabolism in familial hypercholesterolemia. JLipid Res. 2021;62:100062.

25. Ota T, et al. Inhibition of apolipoprotein B100 secretion by lipid-induced hepatic endoplasmic reticulum stress in rodents. JClin Invest. 2008;118(1):316-332.

26. Caviglia JM, et al. Different fatty acids inhibit apoB100 secretion by different pathways: unique roles for ER stress, ceramide, and autophagy. J Lipid Res. 2011;52(9):1636-1651.

27. van de Sluis B, et al. News on the molecular regulation and function of hepatic low-density lipoprotein receptor and LDLR-related protein 1. Curr Opin Lipidol. 2017;28(3):241-247.

28. Brown MS, Goldstein JL. The SREBP pathway: regulation of cholesterol metabolism by proteolysis of a membrane-bound transcription factor. Cell. 1997;89(3):331-340.

29. Chen BP, et al. ATF3 and ATF3 delta Zip. Transcriptional repression versus activation by alternatively spliced isoforms. J Biol Chem. 1994;269(22):15819-15826.

30. Jadhav K, Zhang Y. Activating transcription factor 3 in immune response and metabolic regulation. Liver Res. 2017;1(2):96-102.
31. Gold ES, et al. ATF3 protects against atherosclerosis by suppressing 25-hydroxycholesterol-induced lipid body formation. J Exp Med. 2012;209(4):807-817.

32. Lim JH, et al. Organelle stress-induced activating transcription factor-3 downregulates low-density lipoprotein receptor expression in Sk-Hep1 human liver cells. Biol Chem. 2011;392(4):377-385.

33. Reinke AW, et al. Networks of bZIP protein-protein interactions diversified over a billion years of evolution. Science. 2013;340(6133):730-734.

34. Rodríguez-Martínez JA, et al. Combinatorial bZIP dimers display complex DNA-binding specificity landscapes. Elife. 2017;6:e19272.

35. Pan YX, et al. Activation of the ATF3 gene through a co-ordinated amino acid-sensing response programme that controls transcriptional regulation of responsive genes following amino acid limitation. Biochem J. 2007;401(1):299-307.

36. Xu Y, et al. Hepatocyte ATF3 protects against atherosclerosis by regulating HDL and bile acid metabolism. Nat Metab. 2021;3(1):59-74.

37. Taconic Biosciences, Inc. Trib1 - Model 10265 cKO. https://www.taconic.com/mouse-model/ trib1-cko-10265. Accessed September 29, 2021.

38. Zhang P, et al. Enhancement of hematopoietic stem cell repopulating capacity and self-renewal in the absence of the transcription factor C/EBP alpha. Immunity. 2004;21(6):853-863.

39. Yan $Z$, et al. Human thyroxine binding globulin (TBG) promoter directs efficient and sustaining transgene expression in liver-specific pattern. Gene. 2012;506(2):289-294.

40. Rader DJ, et al. In vivo metabolism of apolipoprotein A-I on high density lipoprotein particles LpA-I and LpA-I,A-II. JLipid Res. 1991;32(11):1849-1859.

41. Borén J, et al. Studies on the assembly of apolipoprotein B-100- and B-48-containing very low density lipoproteins in McA-RH7777 cells. J Biol Chem. 1994;269(41):25879-25888.

42. Patro R, et al. Salmon provides fast and biasaware quantification of transcript expression. Nat Methods. 2017;14(4):417-419.

43. Frankish A, et al. GENCODE reference annotation for the human and mouse genomes. Nucleic Acids Res. 2019;47(d1):D766-D773.

44. Soneson C, et al. Differential analyses for RNAseq: transcript-level estimates improve gene-level inferences. F1000Res. 2015;4:1521.

45. Love MI, et al. Moderated estimation of fold change and dispersion for RNA-seq data with DESeq2. Genome Biol. 2014;15(12):550.

46. Edgar R, et al. Gene Expression Omnibus: NCBI gene expression and hybridization array data repository. Nucleic Acids Res. 2002;30(1):207-210. 